\title{
Efficient Algorithms for Verification of Equivalences for Probabilistic Processes
}

\author{
Linda Christoff Ivan Christoff \\ Department of Computer Systems \\ Uppsala University \\ S-75120 Uppsala, Sweden
}

\begin{abstract}
We present algorithms for automatic verification of equivalences between probabilistic processes. The equivalences we shall verify are testing type equivalences, based on probabilities for probabilistic processes to perform strings of observable events when interacting with different environments (or tests). Our algorithms are based on a recent polynomial-time algorithm for establishing language equivalence of probabilistic automata by Tzeng. We show that the time complexity of our algorithms is polynomial with respect to the number of states in the compared processes.
\end{abstract}

\section{Introduction}

During the last decade several theories for specifying and analysing the behavior of concurrent processes have emerged (e.g. CCS by Milner [Mi 89], CSP by Hoare [Ho 85], and ACP by Bergstra and Klop [BK 84]). These theories, commonly referred to as process algebras, include different notions of equivalence between processes. Several verification methods for establishing equivalences between processes have been developed, and implemented in tools (e.g. see [GLZ 89, CPS 90, Ch 90a]).

In the past couple of years, probabilistic models for specifying and analysing behavior of concurrent processes have been introduced, e.g. see [LS 89, Ch 90b, GJS 90, HJ 90]. For these models, two types of equivalences have been defined: bisimulation type equivalences [LS $89, \mathrm{HJ} 90$ ], and equivalences based on probabilities for processes to perform strings of events [Ch 90b, JS 90].

The work presented in this paper is based on the probabilistic process model described in [Ch 90b, Ch 90c]. We shall present efficient algorithms for verification of three equivalences for probabilistic processes: probabilistic trace equivalence $\left(=_{\mathbb{t}}\right)$, probabilistic failure equivalence $\left(=_{\mathrm{fe}}\right)$, and strong probabilistic test equivalence $\left(=_{\mathrm{ste}}\right)$.

The equivalences we shall consider are based on probabilities for processes to perform strings of observable events, when interacting with different types of tests. Verification of an equivalence between two processes involves showing that these probabilities are equal for all strings of 
observable events and all tests. For recursive processes, verification requires that an infinite number of probabilities are examined. The verification algorithms presented in this paper are based on finding finite subsets of probabilities for the compared processes, whose equality implies equivalence of all probabilities. To find such finite subsets efficiently, we adopt ideas from an algorithm for establishing language equivalence of probabilistic automata by Tzeng [Tz].

The paper is organized as follows: In section 2 we define the probabilistic process model and the equivalences. The verification algorithms are presented in section 3 . The complexity of these algorithms is examined in section 4. In section 5 we summarize the results.

\section{Process Model and Equivalences}

In this section we shall define the probabilistic process model and the equivalences.

\subsection{Probabilistic Processes}

The operational behavior of probabilistic processes is modeled in [Ch $90 \mathrm{~b}, \mathrm{Ch} 90 \mathrm{c}]$, using Plotkin's notion of transition systems [P1 81], extended with probabilities.

Definition 2.1.1 A finite labeled probabilistic transition system (PTS) is a triple, $(S, E, \pi)$, where:

- $S$ is a finite set of states, ranged over by $s, s^{\prime}, s 1, s 2$, etc.

- $E=L \cup\{\tau\}$, where $\tau \notin L$, is a finite set of events (ranged over by $e, e^{\prime}$, etc.), $L$ is a finite set of observable events (ranged over by $a, a^{\prime}$, etc.), and $\tau$ is an $u n$ observable event.

- $\pi: S \times E \times S \rightarrow[0,1]$, is a transition probability function. A requirement for $\pi$ is:

$$
\forall s \in S: \quad \Sigma_{e \in E, s^{\prime} \in S^{\pi}} \pi\left(s, e, s^{\prime}\right)= \begin{cases}1 & \text { if } \exists s^{\prime}: \pi\left(s, e, s^{\prime}\right)>0 \\ 0 & \text { if } \neg \exists s^{\prime}: \pi\left(s, e, s^{\prime}\right)>0\end{cases}
$$

Intuitively, $\pi(s, e, s)$ is the probability for performing event $e$ at state $s$, and moving to state $s^{\prime}$. We shall use $L^{*}$ to denote the set of strings of observable events (ranged over by $\sigma, \sigma^{\prime}$, etc.), $\varepsilon$ to denote the empty string and:

$$
\begin{array}{ll}
s \stackrel{e}{\longrightarrow} s^{\prime} & \text { to denote that } \pi(s, e, s)=\mathrm{p} \\
s \stackrel{e}{\longrightarrow} s^{\prime} & \text { to denote that } \pi(s, e, s)>0 \\
s \stackrel{e}{\longrightarrow} & \text { to denote that } \exists s^{\prime}: \pi(s, e, s)>0 \\
s \stackrel{\tau^{\mathrm{n}}}{\longrightarrow} s^{\prime} & \text { to denote } \stackrel{\tau}{\longrightarrow} s_{1} \stackrel{\tau}{\longrightarrow} \ldots s_{\mathrm{n}-1} \stackrel{\tau}{\longrightarrow} s^{\prime}(\text { where } \mathrm{n} \geq 0) \\
s \stackrel{\tau^{\mathrm{n}} a}{\longrightarrow} & \text { to denote } s \stackrel{\mathfrak{\tau}^{\mathrm{n}}}{\longrightarrow} s^{\prime} \stackrel{a}{\longrightarrow}
\end{array}
$$

Definition 2.1.2 Let $(S, E, \pi)$ be a PTS. A function that computes the states reached in a PTS after performing a string of observable events, After: $2^{S} \times L^{*} \rightarrow 2^{S}$, is defined for all $S^{\prime} \subseteq S, a \in L$ and $\sigma \in L^{*}$, as:

$$
\begin{aligned}
& \text { 1) } \mathcal{A f t e r}\left(S^{\prime}, \varepsilon\right)=\left\{s^{\prime} \in S \mid \exists s \in S^{\prime} . s^{\tau^{\mathrm{n}}} \longrightarrow s^{\prime}\right\} \\
& \text { 2) } \mathcal{A f t e r}\left(S^{\prime}, a \sigma\right)=\mathcal{A} \operatorname{ter}\left(\left(s^{\prime} \in S \mid \exists s \in S^{\prime} . s \stackrel{\tau^{\mathrm{n}} a}{\longrightarrow} s^{\prime}\right\}, \sigma\right)
\end{aligned}
$$


Intuitively, After $(\{\}, \sigma)$ defines the set of states reachable by any number of $\tau$ 's, from the states reached after $\sigma$ is performed from $s$.

Definition 2.1.3 (Probabilistic process) Let $(S, E, \pi)$ be a PTS. We interpret each $s \in S$ as a probabilistic process, defined by $\left(S_{s}, E, \pi_{s}\right)$, where:

- $s$ is the initial state

- $S_{s}=\left\{s^{\prime} \in S \mid \exists \sigma \in L^{*} . s^{\prime} \in \mathcal{A} f \operatorname{ter}(\{s\}, \sigma)\right\}$ defines the states reachable from $s$

- $\pi_{s}: S_{s} \times E \times S_{s} \rightarrow[0,1]$, is defined for all $s^{\prime}, s^{\prime \prime} \in S_{s}$ and $e \in E$ as $\pi_{s}\left(s^{\prime}, e, s^{\prime \prime}\right)=\pi\left(s^{\prime}, e, s^{\prime \prime}\right)$

Figure 2.1.1 gives a graphical representation of probabilistic processes. The processes are displayed in a tree-like style in which 'loops' are represented by a reference to a node. For example, the process $s 1$ has two 'loops' for which the corresponding transitions are $(s I, a, s I)$ and $\left(s I^{\prime}, a, s I^{\prime}\right)$.
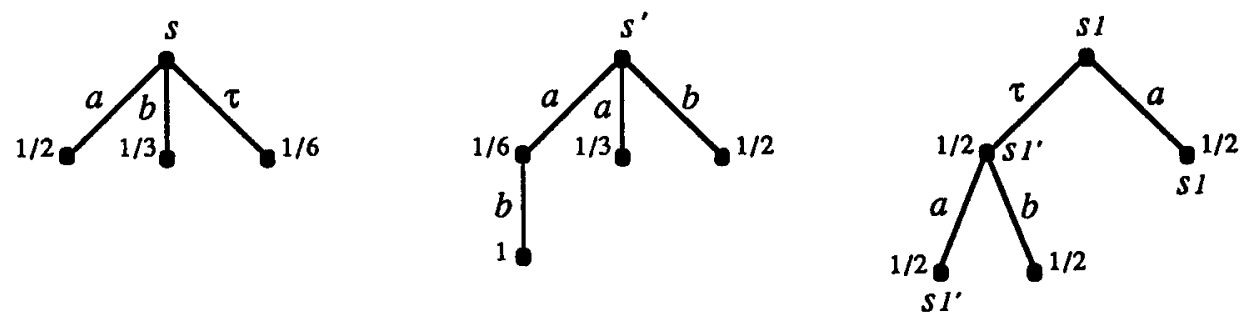

Figure 2.1.1 Probabilistic processes.

\subsection{Equivalences}

In this subsection we define three equivalences for probabilistic processes, based on probabilities for processes to perform strings of observable events when interacting with different types of environments.

First we shall discuss the role of the environment on the transition probabilities in a probabilistic process. When setting the probabilities for the transitions of a process at a state $s$, we assume that all the observable events that can be performed at $s$ are offered by the environment (we also assume that the process can perform $\tau$ 's regardless of which events are offered by the environment). If only a subset of the observable events at $s$ are offered, we normalize the probabilities for the possible transitions at $s$, so that the sum of these probabilities is 1 . The normalization is done in such a manner, so that the relative probabilities between the transitions that can be performed remains the same, as if all observable events were offered. For example, for process $s$ in figure 2.1.1, if only $a$ is offered by the environment, the normalized probabilities for $a$ and $\tau$ would be $3 / 4$ and $1 / 4$, respectively.

Formally, we shall regard the environment of a probabilistic process as a string of sets of observable events, with which the process can interact.

Definition 2.2.1 (Offerings) Let $L$ be a set of observable events. The set of offerings for $L$ is defined as: $\left.O=2^{L}-\{1\}\right\}$. 
To range over $O$ we use $L^{\prime}, L^{\prime \prime}$, etc. $O^{*}$ is used to denote the set of strings of offerings (ranged over by $0,0^{\prime}$, etc.), and $\lambda$ to denote the empty string.

Our equivalences are based on probabilities for processes to perform strings of observable events, when offered strings of offerings. As an example, consider $s I$ in figure 2.1.1. We wish to compute the probability for process $s 1$ to perform the string of observable events $a b$, when $\{a, b\}\{b\}$ is offered. Since $\tau$ 's are unobservable there can be an arbitrary number of $\tau$ 's interleaved in the string (e.g. $\tau^{\mathrm{n}} a \tau^{\mathrm{m}} b$, where $\mathrm{n} \geq 0$ and $\mathrm{m} \geq 0$ ). First we compute the probabilities for performing all $\tau^{\mathrm{n}} a$ strings when $\{a, b\}$ is offered (1/2 for $a$ and $1 / 4$ for $\left.\tau a\right)$, and from the states reached immediately after $a$ and $\tau a$ ( $s I$ and $\left.s l^{\prime}\right)$, we compute the probabilities for performing all $\tau^{\mathrm{m}} b$ strings when $\{b\}$ is offered ( 1 for both $\tau b$ and $b$ ). We then multiply our results to obtain the probabilities for performing $\tau a b$ and $a \tau b$, and sum these probabilities to obtain the probability for performing the string of observable events $a b:(1 / 2) \cdot 1+(1 / 4) \cdot 1=3 / 4$.

To define a function which computes such string probabilities, we need to formalize the notion of 'states reached immediately after a string of observable events is performed'.

Definition 2.2.2 (Just-after states) Let $(S, L \cup\{\tau\}, \pi)$ be a PTS. A function for computing the states reached in a probabilistic process immediately after a string of observable events is performed, Just-after : $2^{S} \times L^{*} \rightarrow 2^{S}$, is defined for all $S^{\prime} \subseteq S, a \in L$ and $\sigma \in L^{*}$, as:

1) Just-after $\left(S^{\prime}, \varepsilon\right)=S^{\prime}$

2) Just-after $\left(S^{\prime}, a \sigma\right)=$ Just-after $\left.\left(s^{\prime} \in S \mid \exists s \in S^{\prime} . s \stackrel{\tau^{\mathrm{n}} a}{\longrightarrow} s^{\prime}\right\}, \sigma\right)$

We can now define a function which computes specific string probabilities (e.g. for process $s I$ in figure 2.1.1, the probability for $s I \stackrel{\tau^{n} a}{\longrightarrow} s I^{\prime}$ when only $\{a\}$ is offered).

Definition 2.2.3 Let $(S, L \cup\{\tau\}, \pi)$ be a PTS. A function that computes probabilities for performing specific strings of events in probabilistic processes restricted by strings of offerings, $Q^{\prime}: S \times O^{*} \times L^{*} \times S \rightarrow[0,1]$, is defined for all $s, s^{\prime} \in S, L^{\prime} \in O, O \in O^{*}, a \in L$ and $\sigma \in L^{*}$, inductively as:

1) $Q^{\prime}\left(s, \lambda, a, s^{\prime}\right)=0$

2) $Q^{\prime}\left(s, 0, \varepsilon, s^{\prime}\right)= \begin{cases}1 & \text { if } s^{\prime}=s \\ 0 & \text { if } s^{\prime} \neq s\end{cases}$

3) $Q^{\prime}\left(s, L^{\prime}, a, s^{\prime}\right)=$ if $\left(a \notin L^{\prime}\right)$ or $\left(s^{\prime} \notin J u s t\right.$-after $\left.(\{\}, a)\right)$ then 0

$$
\text { else } \pi_{L^{\prime}}\left(s, a, s^{\prime}\right)+\Sigma_{s^{\prime \prime} \in \operatorname{Sial}(s)} \pi_{L^{\prime}}\left(s, \tau, s^{\prime \prime}\right) \cdot Q^{\prime}\left(s^{\prime \prime}, L^{\prime}, a, s^{\prime}\right)
$$

4) $Q^{\prime}\left(s, L^{\prime} 0, a \sigma, s^{\prime}\right)=\sum_{\left.s^{\prime \prime} \in \text { gust-after }(s), a\right)} Q^{\prime}\left(s, L^{\prime}, a, s^{\prime \prime}\right) \cdot Q^{\prime}\left(s^{\prime \prime}, 0, \sigma, s^{\prime}\right)$

where $\operatorname{Stat}(s)=\left\{s^{\prime} \in S \mid s \stackrel{\tau}{\longrightarrow} s^{\prime}\right\}$ and $\pi_{L^{\prime}}\left(s, e, s^{\prime}\right)=\frac{\pi\left(s, e, s^{\prime}\right)}{\sum_{e^{\prime} \in L^{\prime} \cup}(\tau), s^{\prime \prime} \in S^{\pi\left(s, e^{\prime}, s^{\prime \prime}\right)}}$

Note that $\pi_{L}$, reflects the normalization procedure (note in particular that due to the conditions in rule $\left.3, \sum_{e^{\prime} \in L^{\prime} \cup(\tau\}, s^{\prime \prime} \in s} \pi\left(s, e^{\prime}, s^{\prime \prime}\right) \neq 0\right)$. For any probabilistic process $s$, we use $Q^{\prime}$ to compute the probability for performing a string $\sigma$ (when offered a string of offerings 0 ), starting at $s$ and terminating at a state $s^{\prime}$ in the set $\left.g_{u s t-a f t e r}(s\}, \sigma\right)$. 
Definition 2.2.4 (String probability) Let $(S, L \cup\{\tau\}, \pi)$ be a PTS. A function that computes probabilities for performing strings of events in probabilistic processes restricted by strings of offerings, $Q: S \times O^{*} \times L^{*} \rightarrow[0,1]$, is defined for all $s \in S, 0 \in O^{*}$ and $\sigma \in L^{*}$, as:

$$
Q(s, 0, \sigma)=\Sigma_{\left.s^{\prime} \in \text { Iust-after }(s), \sigma\right)} Q^{\prime}\left(s, 0, \sigma, s^{\prime}\right)
$$

For two of the equivalences we shall define a function, with which environments that restrict processes to only performing a specific string of events can be defined.

Definition 2.2.5 We define a function that transforms a string of events to a string of offerings, Sets : $L^{*} \rightarrow O^{*}$, for all $\sigma \in L^{*}$, as: 1) $\operatorname{Sets}(\varepsilon)=\lambda$ and 2) $\operatorname{Sets}(a \sigma)=\{a\} \operatorname{Sets}(\sigma)$.

We can now define three equivalences which distinguish processes by probabilities for performing strings of observable events when interacting with: environments that restrict the processes to performing specific strings of events $\left(=_{t \pi}\right)$, environments that restrict the processes to performing specific strings of events followed by any set of events $\left(=_{\mathrm{fe}}\right)$, and any environment $\left(=_{\text {ste }}\right)$. We regard these equivalences as test equivalences, since an environment (i.e. an offering string) can be interpreted as a test.

Definition 2.2.6 (Equivalences) Let $(S, L \cup\{\tau\}, \pi)$ be a PTS. We define, probabilistic trace equivalence $\left(=_{\mathrm{tr}}\right)$, probabilistic failure equivalence $\left(=_{\mathrm{fc}}\right)$ and strong probabilistic test equivalence $\left(=_{\text {ste }}\right)$, for all $s, s^{\prime} \in S$, as:

$$
\begin{array}{llrl}
s={ }_{\mathrm{tr}} s^{\prime} & \Leftrightarrow \forall \sigma \in L^{*}: & Q(s, \operatorname{Sets}(\sigma), \sigma)=Q\left(s^{\prime}, \operatorname{Sets}(\sigma), \sigma\right) \\
s={ }_{\mathrm{fe}} s^{\prime} & \Leftrightarrow \forall L^{\prime} \in O, \sigma \in L^{*}: & \sum_{a \in L^{\prime}} Q\left(s, \operatorname{Sets}(\sigma) L^{\prime}, \sigma a\right)=\sum_{a \in L^{\prime}} Q\left(s^{\prime}, \operatorname{Sets}(\sigma) L^{\prime}, \sigma a\right) \\
s={ }_{\mathrm{ste}} s^{\prime} & \Leftrightarrow \forall 0 \in O^{*}, \sigma \in L^{*}: & Q(s, 0, \sigma)=Q\left(s^{\prime}, 0, \sigma\right)
\end{array}
$$

A set of offering-event string pairs, which characterizes $=_{\text {ste }}$, is defined below.

Definition 2.2.7 (Set of pairs of offering-event strings of equal length) Let $L$ be a nonempty set of observable events, and $O$ the corresponding set of offerings. We define the set of pairs of offering-event strings of equal length, as: $E q=\left\{(0, \sigma) \in O^{*} \times L^{*}|| 0|=| \sigma \mid\right\}$.

Proposition 2.2.1 Let $(S, L \cup\{\tau\}, \pi)$ be a PTS. For any two probabilistic processes $s, s^{\prime} \in S:$

$$
s={ }_{\text {ste }} s^{\prime} \quad \Leftrightarrow \quad \forall(0, \sigma) \in E q: \quad Q(s, 0, \sigma)=Q\left(s^{\prime}, 0, \sigma\right)
$$

Proof See [Ch 91].

\section{Verification Algorithms}

In this section we introduce a matrix representation for the $Q^{\prime}$ values associated with a probabilistic process, adopted from probabilistic automata [Pa 71]. Using this matrix representation we define algorithms for verification of the equivalences presented in section 2 . Our algorithms are based on an algorithm for establishing language equivalence of probabilistic automata by Tzeng [Tz]. The main idea behind the algorithms is, for any two processes, to find a finite set of $Q$ values whose equivalence implies equivalence of all $Q$ values. 


\subsection{Preliminaries}

In this subsection we shall define matrices and vectors associated with a probabilistic process, which can be used to compute $Q$ values. The matrices we shall define contain the $Q$ 'values for a process. Since by the definition of $Q^{\prime}$, a $Q^{\prime}$ value greater than 0 is only possible for the states in a process reached just-after a string of events is performed, it is convenient to have a special notion for these 'just-after states'.

Definition 3.1.1 (Just-after states of a process) Let $(S, L \cup\{\tau\}, \pi)$ be a PTS. For any $s \in S$, we define the finite set $\operatorname{gas}(s) \subseteq S$, as:

$$
\operatorname{Jas}(s)=\left\{s^{\prime} \in S \mid \exists \sigma \in L^{*} . s^{\prime} \in \text { Just-after }(\{s\}, \sigma)\right\}
$$

For notational convenience we shall assume that the states in $g a s(s)$ are renamed as $s_{1}, s_{2}, \ldots$, $s_{\mathrm{n}}$ with $s_{1}=s$ and $\mathrm{n}=|\operatorname{Jas}(s)|$.

We shall now define a matrix which contains the $Q^{\prime}$ values for a probabilistic process for a specific offering-event pair.

Definition 3.1.2 (Matrix of probabilities for an offering-event pair) Let $(S, L \cup\{\tau\}, \pi)$ be a PTS. For all $s \in S, L^{\prime} \in O$ and $a \in L$ we define the matrix:

$$
M^{s}\left(L^{\prime}, a\right)=\left[\mathbf{m}_{\mathrm{ij}}^{s_{\mathrm{ij}}}\left(L^{\prime}, a\right)\right]=\left[Q^{\prime}\left(s_{\mathrm{i}}, L^{\prime}, a, s_{\mathrm{j}}\right)\right]
$$

$M^{s}\left(L^{\prime}, a\right)$ is a square matrix of order $|g a s(s)|$, indexed by the states in $\operatorname{gas}(s)$.

We use definition 3.1.2 to define a matrix of probabilities for a pair of offering-event strings.

Definition 3.1.3 (Matrix of probabilities for a pair of offering-event strings) Let $(S, L \cup\{\tau\}, \pi)$ be a PTS. We define a matrix of probabilities for a pair of offering-event strings, $M^{s}(0, \sigma)$, for all $s \in S$ and $\left(L^{\prime}, a\right),(0, \sigma) \in E q$, as: (1) $M^{s}(\lambda, \varepsilon)=1$ (i.e. $M^{s}(\lambda, \varepsilon)$ is a square unit matrix of order $|\mathrm{gas}(s)|)$ and $(2) M^{s}\left(L^{\prime} 0, a \sigma\right)=M^{s}\left(L^{\prime}, a\right) \cdot M^{s}(0, \sigma)$.

Proposition 3.1.1 Let $(S, L \cup\{\tau\}, \pi)$ be a PTS. For all $s \in S$ and $(0, \sigma) \in E q$ :

$$
\mathbf{m}_{\mathrm{ij}}^{s_{\mathrm{j}}}(0, \sigma)=Q^{\prime}\left(s_{\mathrm{i}}, 0, \sigma, s_{\mathrm{j}}\right)
$$

Proof By induction on the length of $o$ and $\sigma$ (see [Ch 91]).

In order to express the $Q$ values for a process in terms of the previously defined matrices, we need to define two vectors.

Definition 3.1.4 (Final vector). Let $(S, L \cup\{\tau\}, \pi)$ be a PTS. For any $s \in S$, we define a final vector, $\eta^{s}$, as a column vector, with all entries equal to 1 and $\left|\eta^{s}\right|=|\operatorname{gas}(s)|$.

The $\mathrm{i}^{\text {th }}$ row of $\mathrm{M}^{s}(0, \sigma)$ contains the probabilities for performing $\sigma$ when $\mathrm{o}$ is offered, starting at $s_{\mathrm{i}}$ and moving to a state $s_{\mathrm{j}}$ in $g_{a s}(s)$. Since the $\mathrm{i}^{\text {th }}$ entry of the vector $M^{s}(0, \sigma) \cdot \eta^{s}$ is the sum of the entries in the $i^{\text {th }}$ row of $M^{s}(0, \sigma)$, it represents the probability for performing $\sigma$ when $O$ is offered, starting at $s_{\mathrm{i}}$ and moving to any state in $\operatorname{las}(s)$. By definition 3.1.1 we know that $s=s_{1}$. Consequently, $Q(s, 0, \sigma)$ is the first entry in the vector $M^{s}(0, \sigma) \cdot \eta^{s}$. In order to select individual entries from $M^{s}(0, \sigma) \cdot \eta^{s}$ we shall define one more vector. 
Definition 3.1.5 (Initial distribution vector). Let $(S, L \cup\{\tau\}, \pi)$ be a PTS. We define the initial distribution vector, $\psi^{s}$, for any $s \in S$, as: $\psi^{s}=(1,0, \ldots, 0)$, with $|\psi s|=|\operatorname{gs}(s)|$.

Proposition 3.1.2 Let $(S, L \cup\{\tau\}, \pi)$ be a PTS. For all $s \in S$ and $(0, \sigma) \in E q$ :

$$
Q(s, 0, \sigma)=\psi^{s} \cdot M^{s}(0, \sigma) \cdot \eta^{s}
$$

Proof The proof uses the definitions of $Q \psi^{s}, \eta^{s}$ and proposition 3.1.1 (see [Ch 91]).

\subsection{Algorithms for Verification of $=_{\text {ste }}$ and $=_{t r}$}

In this subsection we shall define $=_{\text {ste }}$ and $=_{t r}$ in terms of $\psi, M$ and $\eta$. Using these definitions, we develop verification algorithms for the equivalences. We shall begin with $=_{\text {ste }}$.

Proposition 3.2.1 Let $(S, L \cup\{\tau\}, \pi)$ be a PTS. For any $s, s^{\prime} \in S$ :

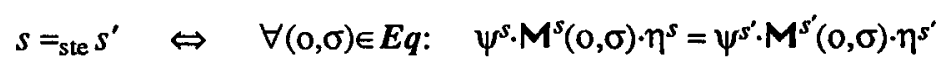

Proof Follows directly from propositions 2.2.1 and 3.1.2.

From proposition 3.2.1 follows that we can verify $=_{\text {ste }}$ between two probabilistic processes $s$ and $s^{\prime}$, by comparing if: $\psi^{s} \cdot M^{s}(0, \sigma) \cdot \eta^{s}=\psi^{s^{\prime}} \cdot M^{s^{\prime}}(0, \sigma) \cdot \eta^{s^{\prime}}$, for all $(0, \sigma) \in E q$. If $s$ and $s^{\prime}$ are recursive processes, there are an infinite number of values to compare. Our objective is to find a finite set of such values, whose equivalence implies that $s=_{\text {ste }} s^{\prime}$. In order to do this we adopt a notion of summation for matrices of probabilities from [Pa 71].

Definition 3.2.1 (Direct sum) Let $(S, L \cup\{\tau\}, \pi)$ be a PTS. For all $s, s^{\prime} \in S$ and $(0, \sigma) \in E q$, we define the direct sum of $M^{s}(0, \sigma)$ and $M^{s}(0, \sigma)$, as:

$$
M^{s+s^{\prime}}(0, \sigma)=\left[\begin{array}{cc}
M^{s}(0, \sigma) & \mathbf{0} \\
\mathbf{0} & M^{s^{\prime}}(0, \sigma)
\end{array}\right]
$$

Note that $M^{s+s^{*}}(0, \sigma)$ is a square matrix of order $|\operatorname{gas}(s)|+|\operatorname{Jas}(s)|$, and that direct sum has the following property: $M^{s+s^{\prime}}\left(o L^{\prime}, \sigma a\right)=M^{s+s^{\prime}}(0, \sigma) \cdot M^{s+s^{\prime}}\left(L^{\prime}, a\right)$.

We shall express $=_{\text {ste }}$ using the direct sum construct.

Proposition 3.2.2 Let $(S, L \cup\{\tau], \pi)$ be a PTS. For any $s, s^{\prime} \in S$ :

$$
s=s_{\text {ste }} s^{\prime} \quad \Leftrightarrow \quad \forall(0, \sigma) \in E q: \quad\left[\psi^{s}, \psi^{s^{\prime}}\right] \cdot M^{s+s^{\prime}}(0, \sigma) \cdot\left[\begin{array}{c}
\eta^{s} \\
-\eta^{s^{\prime}}
\end{array}\right]=0
$$

Proof The proof uses the definition of $\mathrm{M}^{s+s^{\circ}}$ and proposition 3.2.1 (see [Ch 91]).

In (1), $\left[\psi^{s}, \psi^{s^{\prime}}\right] \cdot M^{s+s^{\prime}}(0, \sigma)$, defines a row vector. We shall use the set of all such vectors, $\bigcup_{(0, \sigma) \in E q}\left\{\left[\psi^{s}, \psi^{s}\right] \cdot M^{s+s^{\prime}}(0, \sigma)\right\}$, in the algorithm for verification of $=_{\text {ste }}$.

Definition 3.2.2 Let $(S, L \cup\{\tau\}, \pi)$ be a PTS. We define the $\left(=_{\text {ste }}\right)$-linear space for any two processes $s, s^{\prime} \in S$, as: $\mathcal{L}_{\text {ste }}=\operatorname{span}\left(\cup_{(0, \sigma) \in E_{q}}\left\{\left[\psi^{s}, \psi^{s^{\prime}}\right] \cdot M^{s+s}(0, \sigma)\right\}\right)$, where span is a function, which for a set of vectors $\mathbf{U}$, generates all the linear combinations of the vectors in $\mathbf{U}$. 
Since each $\left[\psi^{s}, \psi^{s^{\prime}}\right] \cdot M^{s+s^{\prime}}(0, \sigma)$ is a row vector with $|\operatorname{gas}(s)|+\left|\operatorname{gas}\left(s^{\prime}\right)\right|$ elements, a basis for $\mathcal{L}_{\text {ste }}$ has at most $|\operatorname{gas}(s)|+\left|\operatorname{gas}\left(s^{\prime}\right)\right|$ vectors. We define $=_{\text {ste }}$ in terms of such a basis.

Lemma 3.2.1 Let $(S, L \cup\{\tau\}, \pi)$ be a PTS. For any two processes $s, s^{\prime} \in S$, if $\boldsymbol{U}$ is a basis for $L_{\text {ste }}$ :

$$
s={ }_{\text {ste }} s^{\prime} \quad \Leftrightarrow \quad \forall v_{\mathrm{i}} \in \mathbf{U}: \boldsymbol{v}_{\mathrm{i}}\left[\begin{array}{c}
\eta^{s} \\
-\eta^{s^{\prime}}
\end{array}\right]=0
$$

Proof The proof uses the fact that $\boldsymbol{U}$ is a basis for $\mathcal{L}_{\text {ste, }}$, and proposition 3.2 .2 (see [Ch 91]).

In order to prove $s=$ ste $s^{\prime}$ one can: (i) find $\boldsymbol{U}$, a basis for $\mathcal{L}_{\text {ste }}$, and (ii) if (2) holds for $\boldsymbol{U}$ then $s={ }_{\text {ste }} s^{\prime}$, otherwise $s \neq_{\text {ste }} s^{\prime}$. We shall now give an algorithm which verifies $=_{\text {ste }}$ in this manner.

Algorithm 1 Let $(S, L \cup\{\tau\}, \pi)$ be a PTS. For any $s, s^{\prime} \in S$, we define an algorithm for verification of $=$ ste as:

(1) $\mathbf{U} \leftarrow\{\}$;

(2) queue $\leftarrow$ vertex $(\lambda, \varepsilon)$;

(3) while queue is not empty do

(4) begin

(5) Take an element vertex $(0, \sigma)$ from queue;

(6) Compute $\left[\psi^{s}, \psi^{s^{\prime}}\right] \cdot M^{s+s^{\prime}}(0, \sigma)$;

(7) if $\left[\psi^{s}, \psi^{s}\right] \cdot M^{s+s^{\prime}}(0, \sigma) \notin \operatorname{span}(\mathrm{U})$ then

(8) begin

(9) For all $L^{\prime} \in O$ and $a \in L^{\prime}$, add vertex $\left(0 L^{\prime}, \sigma a\right)$ to queue;

end;

$\boldsymbol{U} \leftarrow \boldsymbol{U} \cup\left\{\left[\psi^{s}, \psi^{s^{\prime}}\right] \cdot M^{s+s^{*}}(0, \sigma)\right\}$

(13) if for all $\mathbf{U} \in \mathbf{U}, \mathbf{U} \cdot\left[\begin{array}{c}\eta^{s} \\ -\eta^{s^{*}}\end{array}\right]=0$ then return(yes) else return(no);

Algorithm 1 generates a rooted tree. Each vertex in the tree, vertex $(0, \sigma)$, corresponds to a vector $\left[\psi^{s}, \psi^{s^{\prime}}\right] \cdot M^{s+s^{\prime}}(0, \sigma)$ in $\mathcal{L}_{\text {ste }}$. The root of the tree corresponds to the vector $\left[\psi^{s}, \psi^{s^{\prime}}\right] \cdot M^{s+s^{\prime}}(\lambda, \varepsilon)$ (i.e. $\left.\left[\psi^{s}, \psi^{s^{\prime}}\right]\right)$. As the tree grows, a basis for $\mathcal{L}_{\text {ste }}$ is constructed in $\boldsymbol{v}$. A queue is used to keep track of the vertices of the tree that have been created but not 'handled'.

The algorithm works as follows. Initially $\boldsymbol{U}$ is empty, and vertex $(\lambda, \varepsilon)$ is the only element in the queue. A vertex, vertex $(0, \sigma)$, is taken from the queue. If the vector corresponding to vertex $(0, \sigma),\left[\psi^{s}, \psi^{s^{\prime}}\right] \cdot M^{s+s^{\prime}}(0, \sigma)$, is linearly independent w.r.t. the vectors in $\boldsymbol{U}$, it is added to $\boldsymbol{U}$. The tree is expanded from vertex $(0, \sigma)$, with the vertices vertex $\left(0 L^{\prime}, \sigma a\right)$, for all $L^{\prime} \in O$ and $a \in L^{\prime}$. These vertices are added to the queue. If the vector corresponding to vertex $(0, \sigma)$ is linearly dependent on the vectors in $\boldsymbol{U}$, the tree is not expanded from this vertex, i.e. vertex $(0, \sigma)$ becomes a leaf of the tree. The algorithm continues until the tree can not be expanded, i.e. queue is empty.

Theorem 3.2.1 Let $(S, L \cup\{\tau\}, \pi)$ be a PTS. For all probabilistic processes $s, s^{\prime} \in S$, algorithm 1 determines if $s=$ ste $s^{\prime}$.

Proof See [Ch 91]. 
Since the approach for verification of $=_{\mathrm{tr}}$ is the same as the one used for verification of $=_{\text {ste }}$, we shall only indicate the necessary modifications. In analogy to the definition of $\mathcal{L}_{\text {ste }}$, we define a $\left(=_{t}\right)$-linear space, $\mathcal{L}_{t r}$, as:

$$
\mathcal{L}_{\mathrm{tr}}=\operatorname{span}\left(\cup_{\sigma \in L^{*}}\left\{\left[\psi^{s}, \psi^{s^{\prime}}\right] \cdot M^{s+s^{\prime}}(\operatorname{Sets}(\sigma), \sigma)\right\}\right)
$$

and if $\boldsymbol{U}$ is a basis for $\mathcal{L}_{\mathrm{tr}}$, then analogously to lemma 3.2.1 we have that:

$$
s={ }_{\mathrm{U}} s^{\prime} \quad \Leftrightarrow \quad \forall \boldsymbol{v}_{\mathrm{i}} \in \mathbf{U}: \boldsymbol{v}_{\mathrm{i}} \cdot\left[\begin{array}{c}
\eta^{s} \\
-\eta^{s^{\prime}}
\end{array}\right]=0
$$

Algorithm 1 can be easily changed to find a basis for $\mathcal{L}_{\mathbb{t r}}$ by changing line (9) to:

(9) For all $a \in L$, add vertex $(0\{a\}, \sigma a)$ to queue;

The revised algorithm is given in full in [Ch 91].

\subsection{An Algorithm for Verification of $=_{\mathrm{fe}}$}

In this subsection we shall define $=_{\mathrm{fc}}$ in terms of the direct sum construct. Using this definition, we develop a verification algorithm for $=_{\mathrm{fe}}$.

Proposition 3.3.1 Let $(S, L \cup\{\tau\}, \pi)$ be a PTS. For any $s, s^{\prime} \in S$ :

$$
\begin{gathered}
s=\mathrm{fe}_{\mathrm{fe}} s^{\prime} \\
\Leftrightarrow L^{\prime} \in O, \sigma \in L^{*}:\left[\psi^{s}, \psi^{s^{\prime}}\right] \cdot M^{s+s^{\prime}}(\operatorname{Sets}(\sigma), \sigma) \cdot \sum_{a \in L^{\prime}} M^{s+s^{\prime}}\left(L L^{\prime}, a\right) \cdot\left[\begin{array}{c}
\eta^{s} \\
-\eta^{s^{\prime}}
\end{array}\right]=0
\end{gathered}
$$

Proof The proof uses proposition 3.1.2, and the definition of $M^{s+s^{\prime}}$ (see [Ch 91]).

Definition 3.3.1 Let $(S, L \cup\{\tau\}, \pi)$ be a PTS. We define' the $\left(=_{\mathrm{fe}}\right)$-linear space for any two processes $s, s^{\prime} \in S$, as:

$$
\mathcal{L}_{\mathrm{fe}}=\operatorname{span}\left(\bigcup_{\sigma \in L^{*}, L^{\prime} \in O}\left\{\left[\psi^{s}, \psi^{s^{\prime}}\right] \cdot M^{s+s^{\prime}}(\operatorname{Sets}(\sigma), \sigma) \cdot \sum_{a \in L^{\prime}} M^{s+s^{\prime}}\left(L^{\prime}, a\right)\right\}\right)
$$

Since each $\left[\psi^{s}, \psi^{s^{\prime}}\right] \cdot M^{s+s^{\prime}}(\operatorname{Sets}(\sigma), \sigma) \cdot \Sigma_{a \in L^{\prime}} M^{s+s^{\prime}}\left(L^{\prime}, a\right)$ is a row vector with $|\operatorname{gas}(s)|+$ $\left|g_{a s}(s)\right|$ elements, we know that a basis for $\mathcal{L}_{\mathrm{fe}}$ has at most $\left|g_{a s}(s)\right|+\left|g_{a s}\left(s^{\prime}\right)\right|$ vectors. We shall now define $\mathrm{ffe}_{\mathrm{fe}}$ in terms of such a basis.

Lemma 3.3.1 Let $(S, L \cup(\tau), \pi)$ be a PTS. For any two processes $s, s^{\prime} \in S$, if $\boldsymbol{U}$ is a basis for $\mathcal{L}_{\mathrm{fe}}$ :

$$
s=\mathrm{fe}_{\mathrm{fe}} s^{\prime} \quad \Leftrightarrow \quad \forall \mathbf{v}_{\mathrm{i}} \in \mathbf{U}: \quad \mathbf{v}_{\mathrm{i}}\left[\begin{array}{c}
\eta^{s} \\
-\eta^{s^{\prime}}
\end{array}\right]=0
$$

Proof The proof uses the fact that $\boldsymbol{U}$ is a basis for $\mathcal{L}_{\mathrm{fe}}$, and proposition 3.3.1 (see [Ch 91]).

In order to prove $s={ }_{\mathrm{fe}} s^{\prime}$ one can find $\boldsymbol{U}$, a basis for $\mathcal{L}_{\mathrm{fe}}$, and if (4) holds for $\boldsymbol{V}$ then $s=\mathrm{fe}_{\mathrm{e}} s^{\prime}$, otherwise $s \neq_{\mathrm{fe}} s^{\prime}$. We shall now give an algorithm which verifies $=\mathrm{fe}_{\mathrm{fe}}$ in this manner. Consider algorithm 2 . 
Algorithm 2 Let $(S, L \cup\{\tau\}, \pi)$ be a PTS. For any $s, s^{\prime} \in S$, we define an algorithm for verification of $=\mathrm{fe}$ as:

(1) $\mathbf{U} \leftarrow\{\}$;

(2) $\boldsymbol{J}^{\prime} \leftarrow\{\}$;

(3) For all $L^{\prime} \in O$, let queue $\leftarrow \operatorname{vertex}\left(L^{\prime}\right)$;

(4) while queue is not empty do

(5) begin

(6) Take an element vertex $\left(\operatorname{Sets}(\sigma) L^{\prime}\right)$ from queue; Compute $\left[\psi^{s}, \psi^{s^{\prime}}\right] \cdot M^{s+s^{\prime}}(\operatorname{Set} s(\sigma), \sigma) \cdot \sum_{a \in L^{\prime}} M^{s+s^{\prime}}\left(L^{\prime}, a\right)$; if $\left[\psi^{s}, \psi^{s^{\prime}}\right] \cdot M^{s+s^{\prime}}(\operatorname{Sets}(\sigma), \sigma) \cdot \sum_{a \in L^{\prime}} M^{s+s^{\prime}}\left(L^{\prime}, a\right) \notin \operatorname{span}(\mathrm{U})$ then begin

if for some $a \in L, L^{\prime}=\{a\}$ then begin

$$
\boldsymbol{U} \leftarrow \mathbf{U} \cup\left\{\left[\psi^{s}, \psi^{s^{\prime}}\right] \cdot \mathbf{M}^{s+s^{\prime}}(\operatorname{Sets}(\sigma), \sigma) \cdot M^{s+s^{\prime}}(\{a\}, a)\right\} ;
$$

For all $L^{\prime \prime} \in O$, add vertex $\left(\operatorname{Set}(\sigma a) L^{\prime \prime}\right)$ to queue; end; end;$$
\text { else } \mathbf{U}^{\prime} \leftarrow \mathbf{U}^{\prime} \cup\left\{\left[\psi^{s}, \psi^{s^{\prime}}\right] \cdot \mathbf{M}^{s+s^{\prime}}(\operatorname{Sets}(\sigma), \sigma) \cdot \Sigma_{a \in L^{\prime}} \mathbf{M}^{s+s^{\prime}}\left(L^{\prime}, a\right)\right\} \text {; }
$$

$$
\text { if for all } \boldsymbol{U} \in \mathbf{U} \cup \boldsymbol{U}^{\prime}, \boldsymbol{v} \cdot\left[\begin{array}{c}
\eta^{s} \\
-\eta^{s^{\prime}}
\end{array}\right]=0 \text { then return(yes) else return(no); }
$$

Algorithm 2 produces several trees. The root of each tree is a vertex, i.e. vertex $\left(L^{\prime}\right)$ for some $L^{\prime} \in O$. Each vertex in the trees, $\operatorname{vertex}\left(\operatorname{Set}(\sigma) L^{\prime}\right)$, is associated with a vector

$$
\left[\psi^{s}, \psi^{s^{\prime}}\right] \cdot M^{s+s^{\prime}}(\operatorname{Sets}(\sigma), \sigma) \cdot \sum_{a \in L^{\prime}} M^{s+s^{\prime}}\left(L^{\prime}, a\right),
$$

for some $\sigma \in L^{*}$ and $L^{\prime} \in O$. As the trees grow, two sets of vectors, $\boldsymbol{U}$ and $\boldsymbol{U}^{\prime}$, are constructed. In the correctness proof for the algorithm (see [Ch 91]), we show that a basis for $\mathcal{L}_{\mathrm{fe}}$ can be constructed from the union of these two sets of vectors. A queue is used to keep track of the vertices in all trees that have been created but not 'handled'.

The algorithm works as follows. Initially $\boldsymbol{V}$ and $\boldsymbol{V}^{\prime}$ are empty, and for all $L^{\prime} \in O$, vertex $\left(L^{\prime}\right)$ are the only elements in the queue. A vertex, vertex $\left(\operatorname{Sets}(\sigma) L^{\prime}\right)$, is taken from the queue. If the vector corresponding to $\operatorname{vertex}\left(\operatorname{Sets}(\sigma) L^{\prime}\right)$ is linearly independent to the vectors in $\boldsymbol{V}$ and $\left|L^{\prime}\right|=1$ (i.e. $L^{\prime}=\{a\}$ for some $\left.a \in L\right)$, the vector is added to $\boldsymbol{U}$. The tree containing vertex $(\operatorname{Set}(\sigma)\{a\})$ is expanded by adding the vertices vertex $\left(\operatorname{Set}(\sigma a) L^{\prime \prime}\right)$, for all $L^{\prime \prime} \in O$, to the queue. If the vector corresponding to $\operatorname{vertex}\left(\operatorname{Sets}(\sigma) L^{\prime}\right)$ is linearly independent to the vectors in $\mathbf{U}$ and $\left|L^{\prime}\right|>$ 1 , the vector is added to $U^{\prime}$. The tree containing vertex $\left(\operatorname{Sets}(\sigma) L^{\prime}\right)$ is not expanded from this vertex, i.e. vertex $\left(\operatorname{Sets}(\sigma) L^{\prime}\right)$ becomes a leaf. If the vector corresponding to $\operatorname{vertex}\left(\operatorname{Sets}(\sigma) L^{\prime}\right)$ is linearly dependent on the vectors in $\boldsymbol{U}$, the tree containing vertex $\left(\operatorname{Set}(\sigma) L^{\prime}\right)$ is not expanded from this vertex. The algorithm continues until no tree can be expanded, i.e. queue is empty.

Theorem 3.3.1 Let $(S, L \cup\{\tau\}, \pi)$ be a PTS. For all probabilistic processes $s, s^{\prime} \in S$, algorithm 2 determines if $s={ }_{\mathrm{re}} s^{\prime}$.

Proof See [Ch 91]. 


\section{Complexity Analysis}

To determine the complexity of verifying $=_{i}$ (for $i \in\{t r$,fe,ste $\}$ ), we need to consider: $i$ ) the complexity of constructing the matrices required for the verification algorithm for $=_{i}$ and ii) the complexity of the verification algorithm for $=_{i}$.

The complexity of constructing matrices is defined by the number of matrices required times the complexity of constructing each matrix. The number of matrices required (per process) for verification of $=_{t r}$ is $|L|$, and for verification of $=_{\mathrm{fe}}$ and $=_{\text {ste }},\left(2^{|L|}-1\right) \cdot|L|$. Constructing each matrix for a process $s$ involves solving a system of $\left|S_{s}\right|$ linear equations for each column in the matrix. This can be done in $O\left(\left|S_{s}\right|^{2.81}\right)$ time [AHU 74]. Since there are $|g a s(s)|$ columns in each matrix, any matrix can be constructed in $|\operatorname{Jas}(s)| \cdot O\left(\left|S_{s}\right|^{2.81}\right)$ time. Assuming $|L|$ is constant, and observing that $|\operatorname{gas}(s)| \leq\left|S_{s}\right|$, the complexity of constructing the matrices required for verification of any of the equivalences for any two processes $s$ and $s^{\prime}$ is: $O\left(\left|S_{s}{ }^{3.81}+\right| S_{s}||^{3.81}\right)$.

The verification algorithm for $=_{\text {ste }}$ constructs a tree with at most $|\operatorname{gas}(s)|+|J a s(s)|$ internal nodes (i.e. non-leaf nodes), and $\left(|L| \cdot\left(2^{|L|}-1\right)-1\right) \cdot\left(|\operatorname{gas}(s)|+\left|\operatorname{gas}\left(s^{\prime}\right)\right|\right)+1$ leaves [Kn 68]. For each node we must: a) compute the vector associated with the node, and b) determine if it is linearly dependent on the vectors in $\mathbf{U}$.

a) The vector associated with each node can be calculated by multiplying the $\left(|g a s(s)|+\left|\operatorname{gas}\left(s^{3}\right)\right|\right)$-dimensional vector associated with the father of the node with a $M^{s+s^{\prime}}\left(L^{\prime}, a\right)$ matrix, of order $|\operatorname{gas}(s)|+\left|\operatorname{gas}\left(s^{\prime}\right)\right|$. This can be computed in $O\left(\left(|\operatorname{gas}(s)|+\left|\operatorname{gas}\left(s^{\prime}\right)\right|\right)^{2}\right)$ time [Kn 69].

b) Determining linear dependence requires $O\left(\left(|\operatorname{gas}(s)|+\left|\operatorname{gas}\left(s^{2}\right)\right|\right)^{3}\right)$ time $[\mathrm{Kn} 69]$.

The complexity for the verification algorithm for $=_{\text {ste }}$ is consequently:

$$
\left(|\operatorname{gas}(s)|+|\operatorname{gas}(s)|+\left(\left(|L| \cdot\left(2^{|L|}-1\right)-1\right) \cdot(|\operatorname{gas}(s)|+|\operatorname{gas}(s)|)+1\right)\right) \cdot O\left(\left(|\operatorname{gas}(s)|+\left|\operatorname{gas}\left(s^{\prime}\right)\right|\right)^{3}\right)
$$

Assuming that $|\boldsymbol{L}|$ is constant, this becomes: $O\left(\left(|\operatorname{gas}(s)|+\left|\operatorname{gas}\left(s^{2}\right)\right|\right)^{4}\right)$.

The verification algorithms for $=_{\mathrm{tr}}$ and $=_{\mathrm{fc}}$ have the same 'cost' of handling each node as the verification algorithm for $=_{\text {ste }}$. The number of nodes that need to be handled for each of these algorithms is: $(|L|-1) \cdot\left(|\operatorname{Jas}(s)|+\left|\operatorname{gas}\left(s^{\prime}\right)\right|\right)+1$, for $=_{t r}$, and $\left(2^{|L|}-2\right) \cdot\left(|\operatorname{gas}(s)|+\left|\operatorname{gas}\left(s^{\prime}\right)\right|\right)$, for $=_{\mathrm{fe}}$. If $|L|$ is assumed constant, the complexity of both algorithms becomes: $O\left(\left(|\operatorname{Jas}(s)|+\left|\operatorname{Jas}\left(s^{\prime}\right)\right|\right)^{4}\right)$.

If we take into consideration that $|\operatorname{gas}(s)|+\left|\operatorname{gas}\left(s^{\prime}\right)\right| \leq\left|S_{s}\right|+\left|S_{s^{\prime}}\right|$, the complexity of the verification algorithms for $=_{\mathrm{tr}},=_{\mathrm{fe}}$ and $=_{\mathrm{ste}}$ becomes: $O\left(\left(S_{s}|+| S_{s} \mid\right)^{4}\right)$.

\section{Summary of Results}

We have presented algorithms for automatic verification of three equivalences for probabilistic processes. The verification algorithms are based on a matrix representation from probabilistic automata [ $\mathrm{Pa} \mathrm{71]}$, and a polynomial-time algorithm for establishing language equivalence of probabilistic automata by Tzeng [Tz]. 
The time complexity of the verification algorithms is shown to be $O\left(\left(\left|S_{s}\right|+\left|S_{s}\right|\right)^{4}\right)$, where $\left|S_{s}\right|$ and $\left|S_{s}\right|$ represent the number of states for the compared processes. It should be noted that the algorithms for verification of the $=_{\mathrm{tr}}$ and $=_{\mathrm{fe}}$ equivalences for probabilistic processes, stand in contrast to the computationally much harder algorithms for verifying the 'corresponding' equivalences for non-probabilistic processes. For example, for non-deterministic finite state automata, the problems of verifying trace and failure equivalence are known to be PSPACEcomplete [KS 90].

\section{Acknowledgements}

We are grateful to Scott Smolka for drawing our attention to Tzeng's results, to Wen-Guey Tzeng for providing us with [Tz], and to the CAV'91 referees for their helpful comments. This work was in part supported by the Swedish National Board for Technical Development (STU).

\section{References}

[AHU 74] A.V. AHO, J.E. HOPCROFT, J.D. UlLman. The Design and Analysis of Computer Algorithms, Addison-Wesley, 1974.

[BK 84] J.A. BERGSTRA, J.W. KLOP. Process algcbra for synchronous communication. Information and Control 60, pp 109-137, 1984.

[Ch 90a] I. CHRISTOFF. A method for verification of trace and test cquivalence. In Proc. Intl. Workshop on Automatic Verification Methods for Finite State Systems, LNCS 407, pp 81-88, SpringerVerlag, 1990.

[Ch 90b] I. CHRISTOFF. Testing equivalences and fully abstract models for probabilistic processes. In Proc. CONCUR '90, LNCS 458, pp 126-140, Springer-Verlag, 1990.

[Ch 90c] I. ChrIsToff. Testing Equivalences for Probabilistic Processes. PhD thesis, DoCS 90/22, Dept. of Computer Systems, Uppsala University, Sweden, 1990.

[Ch 91] L. CHRISTOFF. Efficient Algorithms for Verification of Equivalences for Probabilistic Processes. Technical report, DoCS 91/28, Dept. of Computer Systems, Uppsala University, Sweden, 1991.

[CPS 90] R. Cleaveland, J. PARRow, B. SteFFen. The concurrency workbench. In Proc. Intl. Workshop on Automatic Verification Methods for Finite State Systems, LNCS 407, pp 24-37, SpringerVerlag, 1990.

[GJS 90] A. GIACALONE, C.-C. JOU, S.A. SMOLKA. Algcbraic reasoning for probabilistic concurrent systems. In Proc. Working Conf. on Programming Concepts and Methods, Sca of Galilec, Isracl, 1990.

[GLZ 89] J.C. GODSKESEN, K.G. LARSEN, M. ZEEBERG. TAV Users Manual. Technical Report R 89-19, Dept. of Mathematics and Computer Science, University of Aalborg, Denmark, 1989.

[HJ 90] H. HANSSON, B. JONSSON. A calculus for communicating systems with time and probabilities. In Proc. 11th IEEE Real-Time Systems Symposium, Orlando, Florida, 1990.

[Ho 85] C.A.R. HOARE. Communicating Sequential Processes. Prentice Hall, 1985.

[JS 90] C.-C. JOU, S.A. SMOLKA. Equivalences, congruences, and complete axiomatizations for probabilistic processes. In Proc. CONCUR '90, LNCS 458, pp 367-383, Springer-Verlag, 1990.

[KS 90] P.C. KANELLAKIS, S.A. SMOLKA. CCS expressions, finite state processes, and three problems of equivalence. Information and Computation 86:1, pp 43-68, 1990.

[Kn 68] D.E. KNUTH. Fundamental Algorithms, Addison-Wesley, 1968.

[Kn 69] D.E. KNUTH. Seminumerical Algorithms, Addison-Weslcy, 1969.

[LS 89] K.G. LARSEN, A. SKou. Bisimulation through probabilistic testing. In Proc. 16th ACM Symp. on Principles of Programming Languages, pp 344-352, 1989.

[Mi 89] R. MILNER. Communication and Concurrency. Prentice Hall, 1989.

[Pa 71] A. PAZ. Introduction to Probabilistic Automata, Academic Press, 1971.

[P1 81] G. PLotKIN. A Structural Approach to Operational Semantics. Technical Report DAIMI FN-19, Computer Science Department, Aarhus University, Aarhus, Denmark, 1981.

[Tz] W.-G. TZENG. A polynomial-time algorithm for the equivalence of probabilistic automata. SIAM Journal on Computing. To appear. 